\title{
Selection Differentials of Component Traits in Truncation Selection for a Proportion-Defined Trait
}

\author{
Masayuki SHOJO and Hiroaki IWAISAKI ${ }^{1}$ \\ Graduate School of Science and Technology, Niigata University, \\ Niigata-shi 950-2181, Japan \\ ' Faculty of Agriculture, Niigata University, \\ Niigata-shi 950-2181, Japan
}

(Received December 10, 1998 ; Accepted February 15, 1999)

\begin{abstract}
In this paper, for one specific type of ratio traits, or a proportion-defined trait (denoted simply as proportion trait), whose numerator trait is contained also in the denominator as its one component, formulae to predict selection differentials for the component traits are derived, assuming upward and downward truncation selection based on phenotypic value of the proportion trait. Two component traits are considered, supposing that these traits follow bivariate normal law and their coefficients of variation are small. The phenotypic changes of component traits are described in terms of a given proportion of population selected, the corresponding truncation point on the proportion trait and phenotypic parameters for the component traits. Using the formulae derived, a deterministic investigation of the selection differentials is conducted for three selected sets of phenotypic parameters for component traits with which the shapes of the distribution of the proportion trait are very nearly symmetric, skewed to the right and skewed to the left. In addition, the signs of the selection differentials are theoretically determined by examining the related inequality including a function of truncation point and the phenotypic parameters. As a consequence, it is revealed that for each of upward and downward selection, there occur three kinds of possible combinations of the signs according to the situations of the phenotypic parameters.
\end{abstract}

Animal Science Journal 70 (3) : 113-117, 1999

Key words : Selection differential, Component trait, Proportion, Truncation selection, Bivariate normality

Characters defined as a proportion such as $\mathrm{x}_{1} /\left(\mathrm{x}_{1}+\right.$ $x_{2}$ ), where $x_{1}$ and $x_{2}$ are quantitative traits, are sometimes considered as a selected trait in livestock improvement. A trait of this type may be called a proportion-defined trait or a proportion trait simply, and is classified as a specific type of ratio traits, as it is obvious from its definition.

Long ago, the statistical and genetic properties of the simplest ratio trait $\mathrm{x}_{1} / \mathrm{x}_{2}$ as a unit character were investigated by applying a log-scale transformation ${ }^{11}$ ) and using, in essence, a Taylor-series expansion ${ }^{10)}$. These approaches can be applied to a ratio function, but do not utilize all information concerning its dis- tributional property. For two correlated normal variates, it is known that their ratio does not follow a normal distribution ${ }^{2,6)}$. The classical theories of quantitative genetics based on the assumption of linearity and normality ${ }^{1)}$ may not be applied simply to non-normal traits such as ratio traits ${ }^{3,7,8)}$, since the superiority of selected individuals and realized response in the progeny depend largely on the distributional property of a character of interest.

Gunset $t^{3,4)}$ showed that the response to direct selection for the simplest ratio trait is complicated by the disproportionate fashion by which selection pressure is exerted on the component traits, and taking account

Corresponding : Hiroaki IWAISAKI (fax : +81 (0) 25-262-6854, e-mail : iwsk@agr. niigata-u.ac.jp)

Anim. Sci. J. 70 (3): 113-117, 1999 
of this fact, he described the expected values in the phenotypic values of the component traits for truncatedly selected individuals. Moreover, Mather et al. ${ }^{8\rangle}$ re-examined Gunsett's description to provide the correct signs of selection differentials of component traits for upward and downward selection.

Following these studies, for selection on the proportion trait, Shojo and Iwaisaki ${ }^{9}$ investigated an expression for truncation point under the assumption that phenotypic values of component traits are normally distributed. In this paper, we further examine selection differentials of the component traits expected from truncation selection on the proportion trait.

\section{Materials and Methods}

We defined a proportion trait, as follows

$$
r=x_{1} /\left(x_{1}+x_{2}\right) \text {, }
$$

where $x_{i}(i=1,2)$ are phenotypic values of the component traits. Herein we assumed that $x_{i}$ follow a bivariate normal distribution with means $\mu_{\mathrm{i}}$, standard deviations $\sigma_{1}$, covariance $\sigma_{12}$, and correlation $\rho$. Then, we first examined expressions for selection differentials of component traits, assuming upward and downward truncation selection on $r$.

When supposed upward selection, for instance, the expected values for component traits in the selected population can be written by the standard approach as

$$
\begin{aligned}
\mu_{i}^{*} & =E\left\{x_{i} \mid r>z\right\} \\
& =p^{-1} \int_{R} \int x_{i} f\left(x_{1}, x_{2}\right) d x_{1}, d x_{2},
\end{aligned}
$$

with the bivariate normal probability density function

$$
\begin{aligned}
& \mathrm{f}\left(\mathrm{x}_{1}, \mathrm{x}_{2}\right)=\left\{2 \pi \sigma_{1} \sigma_{2}\left(1-\rho^{2}\right)^{1 / 2}\right\}^{-1} \exp [-0.5(1 \\
& \left.-\rho^{2}\right)^{-1}\left[\left\{\sigma_{2}^{-1}\left(\mathrm{x}_{1}-\mu_{1}\right)\right\}^{2}-2 \rho\left(\sigma_{1} \sigma_{2}\right)^{-1}\left(\mathrm{x}_{1}-\mu_{1}\right)\right.
\end{aligned}
$$

$$
\left.\left.\left(\mathrm{x}_{2}-\mu_{2}\right)+\left\{\sigma_{2}^{-1}\left(\mathrm{x}_{2}-\mu_{2}\right)\right\}^{2}\right]\right]
$$

where $p$ is the proportion of population selected truncatedly, $z$ is the truncation point in the distribution of $r$, and $R$ is the appropriate region of integration. As described by Shojo and Iwaisaki ${ }^{9)}, \mathrm{z}$ is

$$
\begin{aligned}
& {\left[\mu_{1}\left(\mu_{1}+\mu_{2}\right)-\mathrm{t}^{2}\left(\sigma_{1}^{2}+\sigma_{12}\right)+\alpha \beta\left\{\left(\mu_{1} \mu_{2}-\mathrm{t}^{2} \sigma_{12}\right)^{2}-\right.\right.} \\
& \left.\left.\left(\mu_{1}^{2}-\mathrm{t}^{2} \sigma_{1}^{2}\right)\left(\mu_{2}^{2}-\mathrm{t}^{2} \sigma_{2}^{2}\right)\right\}^{1 / 2}\right] /\left\{\left(\mu_{1}+\mu_{2}\right)^{2}-\mathrm{t}^{2}\left(\sigma_{1}^{2}+\sigma_{2}^{2}\right.\right. \\
& \left.\left.+2 \sigma_{12}\right)\right\},
\end{aligned}
$$

where $t$ is the value of truncation point in the standard normal distribution, given the specified value of $\mathrm{p}, \alpha$ takes +1 and -1 for upward and downward selection, respectively, and $\beta=+1$ if $\mathrm{p}<0.5$ and $\beta=-1$ if $\mathrm{p}>0.5$. When $\mathrm{p}=0.5, \mathrm{z}$ is $\mu_{1} /\left(\mu_{1}+\mu_{2}\right)$, since $\mathrm{t}=0$.

Equation (2) was evaluated by employing two transformations in turn. First, $x_{i}$ were transformed to the standardized normal variates $u_{i}$, where the Jacobian of the transformation was $\sigma_{1} \sigma_{2}$. The second transformation was then $v_{1}=u_{1}$ and $v_{2}=\left(1-\rho^{2}\right)^{-1 / 2}$ $\left(\mathrm{u}_{2}-\rho \mathrm{v}_{1}\right)$, having $\left(1-\rho^{2}\right)^{1 / 2}$ as the Jacobian of the transformation. Consequently, for $\mu_{i}^{*}$, we had

$$
\begin{aligned}
\mu_{1}^{*}= & (2 \pi \mathrm{p})^{-1} \mu_{1} \int_{\mathrm{R}} \int \exp \left\{-0.5\left(\mathrm{v}_{1}^{2}+\mathrm{v}_{2}^{2}\right)\right\} \mathrm{dv}_{1} \mathrm{dv}_{2} \\
& +(2 \pi \mathrm{p})^{-1} \sigma_{1} \int_{\mathrm{R}} \int \mathrm{v}_{1} \exp \left\{-0.5\left(\mathrm{v}_{1}^{2}+\mathrm{v}_{2}^{2}\right)\right\} \\
& \mathrm{dv}_{1} \mathrm{dv}_{2},
\end{aligned}
$$

where the region of integration $\mathbf{R}$ was given as

$$
\begin{aligned}
& \left(\mu_{1}+\sigma_{1} \mathrm{v}_{1}\right) /\left[\mu_{2}+\sigma_{2}\left\{\rho \mathrm{v}_{1}+\left(1-\rho^{2}\right)^{1 / 2} \mathrm{v}_{2}\right\}\right]>\mathrm{z} /(1 \\
& -\mathrm{z}) .
\end{aligned}
$$

The corresponding expression for $\mu_{2}^{*}$ was obtained similarly, performing the transformation $v_{1}=\left(1-\rho^{2}\right.$ )$^{-1 / 2}\left(u_{1}-\rho v_{2}\right)$ and $v_{2}=u_{2}$, where the region $R$ was yielded as

$$
\begin{aligned}
& {\left[\mu_{1}+\sigma_{1}\left\{\left(1-\rho^{2}\right)^{1 / 2} \mathbf{v}_{\mathrm{i}}+\rho \mathbf{v}_{2}\right\}\right] /\left(\mu_{2}+\sigma_{2} \mathbf{v}_{2}\right)>z / /(1} \\
& -\mathrm{z}) .
\end{aligned}
$$

Table 1. Parameter sets considered

\begin{tabular}{llll} 
& \multicolumn{3}{c}{ Set } \\
\cline { 2 - 4 } & \multicolumn{1}{c}{ I } & I & II \\
\hline Trait $\mathrm{x}_{1}$ & & & \\
$\quad$ Population mean $\left(\mu_{1}\right)$ & 1.0 & 1.0 & 2.5 \\
$\quad$ Phenotypic standard deviation $\left(\sigma_{1}\right)$ & 0.05 & 0.05 & 0.5 \\
$\quad$ Trait $\mathrm{x}_{2}$ & & & \\
$\quad$ Population mean $\left(\mu_{2}\right)$ & 1.0 & 2.5 & 1.0 \\
$\quad$ Phenotypic standard deviation $\left(\sigma_{2}\right)$ & 0.05 & 0.5 & 0.05 \\
$\quad$ Phenotypic correlation $(\rho)$ & 0.5 & 0.5 & 0.5 \\
\hline
\end{tabular}


Thus, further carrying out the required integration by the standard procedure and finally calculating $\mu_{i}^{*}-$ $\mu_{\mathrm{i}}$, selection differentials of component traits were derived in terms of phenotypic parameters for component traits.

Next, using the resulting expressions, patterns of selection differentials of component traits were evaluated deterministically for three selected populations with different combinations of the parameter values for component traits. The parameter sets considered (denoted as sets I, II and III), taken from Shojo and Iwaisaki ${ }^{9)}$, are listed in Table 1 . With sets I, II and III, the shape of distribution of the proportion trait was very nearly symmetric, skewed to the right, and skewed to the left, respectively. Both the cases of upward and downward selection were examined, and a total of five levels for the proportion of population selected were considered.

\section{Results and Discussion}

Selection differentials for component trait i $(\mathrm{i}=1$, $2)$, or $\mu_{\mathrm{i}}^{*}-\mu_{\mathrm{i}}$, was obtained as

$$
\begin{aligned}
& \Delta \mathrm{s}_{\mathrm{i}}=\alpha \varepsilon_{\mathrm{i}} \sigma_{\mathrm{i}} \mathrm{p}^{-1}\left\{2 \pi\left(\gamma_{\mathrm{i}}^{2}+1\right)\right\}^{-1 / 2} \exp \left\{-0.5\left(\gamma_{\mathrm{i}}^{2}+\right.\right. \\
& \left.1)^{-1} \delta_{\mathrm{i}}^{2}\right\},
\end{aligned}
$$

where

$$
\begin{aligned}
& \gamma_{1}=\left\{z \sigma_{2}\left(1-\rho^{2}\right)^{1 / 2}\right\} /\left\{(1-z) \sigma_{1}-z \sigma_{2} \rho\right\}, \\
& \delta_{1}=\left\{z \mu_{2}-(1-z) \mu_{1}\right\} /\left\{(1-z) \sigma_{1}-z \sigma_{2} \rho\right\},
\end{aligned}
$$

and $\varepsilon_{1}$ is equal to 1 if $(1-z) \sigma_{1}>z \sigma_{2} \rho$ and to -1 if not, and

$$
\begin{aligned}
& \gamma_{2}=\left\{(1-\mathrm{z}) \sigma_{1}\left(1-\rho^{2}\right)^{1 / 2}\right\} /\left\{\mathrm{z} \sigma_{2}-(1-\mathrm{z}) \sigma_{1} \rho\right\} \\
& \delta_{2}=\left\{(1-\mathrm{z}) \mu_{1}-\mathrm{z} \mu_{2}\right\} /\left\{\mathrm{z} \sigma_{2}-(1-\mathrm{z}) \sigma_{1} \rho\right\},
\end{aligned}
$$

and $\varepsilon_{2}$ equals -1 if $z \sigma_{2}>(1-z) \sigma_{1} \rho$ and equals 1 if not.

Selection differentials for component traits, which were predicted using the derived formulae, are presented in Table 2 for the three selected sets of the parameters for component traits. These values are expressed in terms of the corresponding phenotypic standard deviation for each component trait, which will be denoted as $\Delta s_{i}^{*}$ and clearly show how selection pressure is placed on each of component traits.

For set $I$, in the case of upward selection, the standardized selection differentials $\Delta \mathrm{s}_{\mathrm{i}}^{*}$ were positive for the component trait 1 , or the numerator trait, and negative for the trait 2 which was included only in the denominator. With downward selection, a reverse pattern of the signs was found. Specifically, for this parameter set, absolute values of $\Delta s_{1}^{*}$ and $\Delta s_{2}^{*}$ for upward selection were equal to those of $\Delta \mathrm{s}_{2}^{*}$ and $\Delta \mathrm{s}_{1}^{*}$ for downward selection, respectively, and with $\mathrm{p}=0.5$, absolute values of the selection differentials expected

\begin{tabular}{|c|c|c|c|c|c|c|c|}
\hline \multirow{2}{*}{ Selection } & \multirow{2}{*}{$\begin{array}{l}\text { Proportion } \\
\text { selected } \\
(\%)\end{array}$} & \multicolumn{2}{|c|}{ Set I } & \multicolumn{2}{|c|}{ Set II } & \multicolumn{2}{|c|}{ Sct III } \\
\hline & & $\Delta s_{i}^{*}$ & $\Delta s_{i}^{*}$ & $\Delta s_{i}^{*}$ & $\Delta \mathrm{s}_{2}^{*}$ & $\Delta \mathbf{s}_{i}^{\prime}$ & $\Delta s_{2}^{\prime}$ \\
\hline \multirow[t]{5}{*}{ Upward } & 1 & 1.0933 & -1.5583 & -1.0207 & -2.6425 & 2. 4994 & 0.4484 \\
\hline & 5 & 0.9015 & -1.1560 & -0.7269 & -2.0352 & 1.9569 & 0.4137 \\
\hline & 10 & 0.7918 & -0.9605 & -0.5896 & -1.7263 & 1.6744 & 0.3820 \\
\hline & 50 & 0.3989 & -0.3989 & -0.2213 & -0.7745 & 0.7745 & 0.2213 \\
\hline & 90 & 0.1067 & -0.0880 & -0.0424 & -0.1860 & 0.1918 & 0.0655 \\
\hline \multirow[t]{5}{*}{ Downward } & 1 & -1.5583 & 1.0933 & 0.4484 & 2. 4994 & -2.6425 & -1.0207 \\
\hline & 5 & -1.1560 & 0.9015 & 0.4137 & 1. 9569 & -2.0352 & -0.7269 \\
\hline & 10 & -0.9605 & 0.7918 & 0.3820 & 1.6744 & -1.7263 & -0.5896 \\
\hline & 50 & -0.3989 & 0.3989 & 0.2213 & 0.7745 & -0.7745 & -0.2213 \\
\hline & 90 & -0.0880 & 0.1067 & 0.0655 & 0.1918 & -0.1860 & -0.0424 \\
\hline
\end{tabular}
were found to be the same for all the cases, regardless of type of selection. When $p<0.5$ and $p>0.5$, however, $\Delta s_{i}^{*}$ was lower and higher in absolute value than $\Delta s_{2}^{*}$ for upward selection, respectively, and vice versa

Table 2. Standardized selection differentials of component traits ${ }^{1)}$ for three parameter sets

1) $\Delta \mathrm{s}_{\mathrm{i}}^{*}=$ standardized selection differential of component trait $\mathrm{i}$. 
SHOJO and IWAISAKI

Table 3. Signs of selection differentials of component traits ${ }^{1)}$

\begin{tabular}{ccc}
\hline Condition & Upward selection $(\alpha=+1)$ & Downward selection $(\alpha=-1)$ \\
\hline$\rho<(1-\mathrm{z}) \sigma_{1} / \mathrm{z} \sigma_{2}\left(\varepsilon_{1}=+1\right)$ & $\Delta \mathrm{s}_{1}>0$ & $\Delta \mathrm{s}_{1}<0$ \\
$\rho>(1-\mathrm{z}) \sigma_{1} / \mathrm{z} \sigma_{2}\left(\varepsilon_{1}=-1\right)$ & $\Delta \mathrm{s}_{1}<0$ & $\Delta \mathrm{s}_{1}>0$ \\
$\rho>\mathrm{z} \sigma_{2} /(1-\mathrm{z}) \sigma_{1}\left(\varepsilon_{2}=+1\right)$ & $\Delta \mathrm{s}_{2}>0$ & $\Delta \mathrm{s}_{2}<0$ \\
$\rho<\mathrm{z} \sigma_{2} /(1-\mathrm{z}) \sigma_{1}\left(\varepsilon_{2}=-1\right)$ & $\Delta \mathrm{s}_{2}<0$ & $\Delta \mathrm{s}_{2}>0$ \\
\hline
\end{tabular}

1) $\Delta \mathrm{s}_{\mathrm{i}}=$ selection differential of component trait $\mathrm{i}$.

$\sigma_{\mathrm{i}}=$ phenotypic standard deviation for component trait $\mathrm{i}$.

$\rho=$ phenotypic correlation between component traits.

$z=$ truncation point on proportion trait.

for downward selection. The shape of the distribution of $\mathbf{r}$ with set $\mathbf{I}$ is almost symmetrical, but strictly speaking, those in the upper and lower tails are slightly different from each other ${ }^{9)}$, following that selection pressure put on $r$ acts on component traits by a disproportionate fashion.

In the case of set II with which the distribution of $\mathrm{r}$ is skewed to the right, though with $\mathrm{p}=0.5$, the absolute values of selection differentials in one component trait for two directions of selection were also equal to each other, it was found that direct selection on $\mathrm{r}$, regardless of direction of selection, places the majority of selection pressure on the trait 2 , or the component trait with higher standard deviation. Both of $\Delta s_{1}^{*}$ and $\Delta s_{2}^{*}$ resulting from upward selection were expected to be negative, but the absolute value for the trait 1 was relatively smaller, given a proportion selected. Therefore, for the shape of the distribution of this type, it is seen that a positive selection differential for $r$ is accomplished by differentially decreasing the means of component traits. In contrast, downward selection under set II led to increased means for the two component traits. However, the increment of the mean for the trait 2 was expected to be larger than that for the trait 1 , or the numerator trait, which gives a negative phenotypic response in the proportion trait.

For each of the directions of selection, the third combination of signs for $\Delta s_{1}^{*}$ and $\Delta s_{2}^{*}$ was found with set III. For upward selection, being different from the two upward cases with set I and II, the signs of $\Delta$ $s_{1}^{*}$ and $\Delta s_{2}^{*}$ were both positive for all considered proportions selected, and $\Delta s_{i}^{*}$ was always higher than $\Delta$ $s_{2}^{*}$, given a proportion selected. With downward selection, selection differentials for component traits were both in the negative sign, showing relatively large phenotypic response for the trait 1 with higher standard deviation.

The numerical results given in Table 2 have demonstrated that with the three selected sets of the phenotypic parameters for component traits, there are three possible patterns of the combination of signs for $\Delta s_{\mathrm{i}}$ for each of upward and downward selection on $\mathrm{r}$. Using the terms $\alpha$ and $\varepsilon_{\mathrm{i}}$, these findings may be confirmed theoretically. For instance, $\Delta s_{1}>0$ is given, when $\alpha$ and $\varepsilon_{1}$ are 1 or when both of these terms are -1. The conditions corresponding to the former case are given as $(1-\mathrm{z}) \sigma_{1}>\mathrm{z} \sigma_{2} \rho$, equivalently $\rho<(1$ $-\mathrm{z}) \sigma_{1} /\left(\mathrm{zO}_{2}\right)$, and upward selection. For all combinations of $\alpha$ and $\varepsilon_{\mathrm{i}}$, signs of the selection differentials are summarized in Table 3. However, note that the patterns of $\Delta \mathrm{s}_{1}<0$ and $\Delta \mathrm{s}_{2}>0$ for upward selection and of $\Delta s_{1}>0$ and $\Delta s_{2}<0$ for downward selection do never occur, since $-1 \leq \rho \leq 1$. Moreover, with $\rho \leq 0$, it is found that $\Delta s_{1}>0$ and $\Delta s_{2}<0$, and $\Delta s_{1}<0$ and $\Delta s_{2}>0$ occur in the upward and downward selection, respectively. On the other hand, if $\rho>0$, then it is also possible that phenotypic changes for component traits are both positive or wholly negative. That is, for upward selection, both of $\Delta s_{1}$ and $\Delta s_{2}$ are expected to be in the positive sign, if $1 / \mathrm{q}<\rho<\mathrm{q}$, and be in the negative sign, if $q<\rho<1 / \mathrm{q}$, where $\mathrm{q}=(1-\mathrm{z})$ $\sigma_{1} /\left(z \sigma_{2}\right)$. In contrast, for downward selection, we have $\Delta \mathrm{s}_{1}>0$ and $\Delta \mathrm{s}_{2}>0$ with $\mathrm{q}<\rho<1 / \mathrm{q}$ and $\Delta \mathrm{s}_{1}<$ 0 and $\Delta \mathrm{s}_{2}<0$ with $1 / \mathrm{q}<\rho<\mathrm{q}$. Hence, it is seen that the sign and the relative magnitude of phenotypic 
correlation between component traits play an important role in determining signs of selection differentials for component traits considered in the proportion trait, and that correspondingly, for the case of $\rho>0$, the signs of the selection differentials are dependent on the type of distribution of $\mathbf{r}$.

In this study, for the proportion-based truncation selection, it was revealed that the directions of the phenotypic changes of component traits are automatically determined by the situation of phenotypic parameters for these traits. It was found that selection pressure put on each component trait is obviously disproportionate. Moreover, it was noticed that according to the situation of phenotypic parameters for component traits, for each of upward and downward selection, there occur three combinations of the signs of selection differentials of these traits. The aspect of the genetic changes, which has more interest for us, will be considered in another paper.

\section{References}

1) Falconer DS. Introduction to quantitative genetics. 3 rd ed. 187-208. Longman. London. 1989.

2) Fieller EC. The distribution of the index in a normal bivariate population. Biometrika, 24 : 428-440. 1932.

3) Gunsett FC. Linear index selection to improve traits defined as ratios. Journal of Animal Science, 59 :
1185-1193. 1984.

4) Gunsett FC. Problems associated with selection for traits defined as a ratio of two component traits. In : Proceedings of the Third World Congress on Genetics Applied to Livestock Production, XI : 437-442. 1986.

5) Gunsett FC. Merit of utilizing the heritability of a ratio to predict the genetic change of a ratio. Journal of Animal Science, 65 : 936-942. 1987.

6) Hinkley DV. On the ratio of two correlated normal random variables. Biometrika, 56:635-639. 1969.

7) Iwaisaki H, Wilton JW. Regression of genotypic on phenotypic value of a ratio-defined character. Biometrics, $49: 1154-1163.1993$.

8) Mather DE, Gunsett FC, Allen OB, Kannenberg LW. Estimation of phenotypic selection differentials for predicting genetic responses to ratio-based selection. Genome, $30: 838-843.1988$.

9) Shojo $\mathbf{M}$, Iwaisaki $\mathbf{H}$. Truncation point in truncation selection for a proportion-defined trait. Animal Science and Technology (Jpn.), $69: 417-423.1998$.

10) Sutherland TM. The correlation between feed efficiency and rate of gain, a ratio and its denominator. Biometrics, $21: 739-749.1965$.

11) Turner HN. Ratios as criterion for selection in animal or plant breeding with particular reference to efficiency of feed conversion in sheep. Australian Journal of Agricultural Research, 10 : 565-580. 1959. 